\title{
Estimating Exposure to Traffic-Related Air Pollution and Its Consequences on Respiratory Health in Population Working or Living along the Trunk Road: A Systematic Review
}

\author{
Parfait Houngbégnon ${ }^{1,2,3}$, Eloïc Atindegla3 ${ }^{3}$, Hervé Lawin'2, Victoire Agueh1 \\ ${ }^{1}$ Institut Régional de Santé Publique Comlan Alfred Quenum, University of Abomey-Calavi, Ouidah, Bénin \\ ${ }^{2}$ EcoHealth Chair, Faculty of Health Sciences, University of Abomey-Calavi, Abomey-Calavi, Bénin \\ ${ }^{3}$ Institut de Recherche Clinique du Bénin, Abomey-Calavi, Bénin \\ Email: parfaith2016@gmail.com
}

How to cite this paper: Houngbégnon, P., Atindegla, E., Lawin, H. and Agueh, V. (2020) Estimating Exposure to Traffic-Related Air Pollution and Its Consequences on Respiratory Health in Population Working or Living along the Trunk Road: A Systematic Review. Open Journal of Air Pollution, 9, 61-76.

https://doi.org/10.4236/ojap.2020.94005

Received: September 12, 2020

Accepted: December 1, 2020

Published: December 4, 2020

Copyright $\odot 2020$ by author(s) and Scientific Research Publishing Inc. This work is licensed under the Creative Commons Attribution International License (CC BY 4.0).

http://creativecommons.org/licenses/by/4.0/

\begin{abstract}
Introduction: Urban Air pollution is increasingly becoming a major health and sustainable development issue. Several studies showed that Traffic-related air pollution (TRAP) is one of the main sources of urban air pollution and has serious consequences on respiratory health. As no systematic review focused on the traffic-related air pollution and respiratory health in the target population of individuals working in a shop or in an office or individuals living along the trunk road, the authors conducted the current study to try to fill this gap. Methods: A systematic review search was conducted using MEDLINE (PubMed), Scientific Research Publishing: SCIRP, Web of Science, Google scholar. Studies were included if they meet the following selection criteria: 1) focus on population working or living along a major/trunk road; 2) studies had reported clearly at least on the exposure variables related to TRAP; 3) the association between TRAP and development of respiratory symptoms or respiratory diseases was established. Results: 13 articles were selected on the 192 articles that were retrieved in the initial research. Exposure to traffic-related air pollution was determined by using distance to road, traffic intensity and pollutants measured. The main respiratory health problems found were cough, wheeze, asthma and bronchitis. No article discussed about roundabouts in characterizing exposure to traffic-related air pollution. Conclusion: Distance to road, traffic density and pollutants measured are the usual methods to characterize the exposure to traffic-related air pollution and its consequences on respiratory health. Regarding the context of area occupations in African cities, it is necessary to focus on population around roundabouts and see if they are not more exposed to TRAP.
\end{abstract}


Keywords

TRAP, Urban Air Pollution, Respiratory Health, Trunk Road

\section{Introduction}

Urban Air pollution is increasingly becoming a major health and sustainable development issue. Compared to all other forms of environmental pollution, air pollution causes the largest number of environmental pollution-related deaths [1]. Ninety-two per cent of the world's population is living in places where levels exceed recommended limits [2]. The World Health Organization (WHO) estimates that ambient (outdoor) air pollution was estimated to cause 4.2 million premature deaths worldwide per year in 2016 [3]. Traffic-related air pollution (TRAP) is one of the main sources of urban air pollution [4] [5] [6] [7] [8]. In major African cities, the air quality has deteriorated significantly. In a study conducted from 2015 to 2017 in Benin and Côte d'Ivoire, 24-hour $\mathrm{PM}_{2.5}$ concentrations were found to consistently exceed WHO guidelines [9]. In Ethiopia, Kenya and Uganda, PM pollution levels were estimated to have increased by $182 \%, 162 \%$ and $62 \%$ respectively since the 1970 s to the current period [10]. The increase in number of obsolete vehicles [11], the urbanization plan that does not keep up with the rapid population growth and the rural exodus are among the main reasons for this situation.

The relationship between TRAP exposure and respiratory morbidity has been studied worldwide [8] [12] [13] [14] [15] [16] and several systematic reviews have examined the relationship between exposure to traffic-related air pollution and respiratory health [17]-[23]. But to the author's knowledge, there were not enough systematic reviews that focused on the target population of individuals working or living along the trunk road. The current review is an attempt to fill this gap. The first step will be to take stock of the different approaches used to estimate the exposure of this target group to traffic-related air pollution and to identify the different pollutants that have been studied. Secondly, the different respiratory health problems are identified.

The aim of this review is to examine if the different approaches used to estimate exposure to traffic-related air pollution and its consequences on respiratory health fully respond to the context of West Africa's cities such as Cotonou.

\section{Methodology}

This systematic review adhered to the recommendations outlined in the PRISMA (Preferred Reporting Items for Systematic Reviews and Meta-Analyses) statement [24].

\subsection{Data Sources and Search Strategy}

Searches were performed using a systematic literature search strategy with the 
removal of duplicates, identifying the available data on the topic. The following electronic scientific databases were primarily searched: MEDLINE (PubMed), Scientific Research Publishing: SCIRP (Open Journal of Air Pollution; Open Journal of Respiratory Diseases), Web of Science. Data sources such as the Google Scholar, search on internet for available grey literature, the table of contents of Environmental Health Perspectives and Atmospheric Environment were searched. The authors also hand-searched references list from included studies in order to found any relevant titles. Medical search terms (MeSH) and keywords such as "traffic" AND ("Air pollution" OR "TRAP") AND ("respiratory symptoms" OR "respiratory diseases") AND ("major road" OR "trunk road" OR "main road" OR "busy road" OR "main street" OR "major street") were used as combinations of text or thesaurus terms to conduct search in the various databases, with the aim of identifying eligible articles for inclusion in the review.

\subsection{Studies Selection}

All original studies, published in English or French including observational or epidemiological studies (cross-sectional, cohort, case-control studies) published from inception January 2000 to December 2019, which discussed about traffic-related air pollution and respiratory symptoms in population working or living along major or trunk roads were eligible for this review. The authors have only included those studies that mentioned clearly the exposure variable addressed. Another inclusion criterion was that the article should address the various respiratory symptoms or respiratory diseases that were associated with traffic-related air pollution.

These different selection criteria concerning specifically the type of study and all expected characteristics of the results are clearly summarized and detailed in Table 1.

Table 1. Selection criteria.

\begin{tabular}{|c|c|}
\hline Inclusion criteria & Exclusion criteria \\
\hline 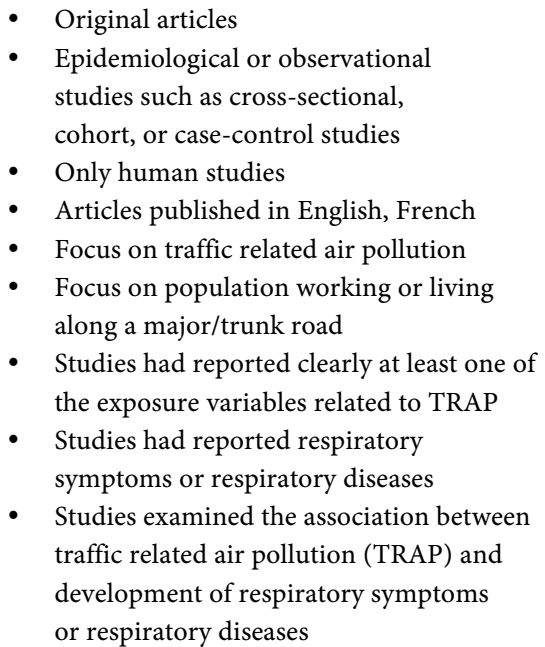 & $\begin{array}{l}\text { - Non-research studies, off-topic } \\
\text { studies, studies concerning } \\
\text { populations not exclusively exposed } \\
\text { to traffic-related air pollution } \\
\text { - } \quad \text { Review, commentaries } \\
\text { - Animal studies } \\
\text { - Abstract or full articles not available } \\
\text { - Studies about TRAP but not on } \\
\text { major/trunk road } \\
\text { - Studies that measured exclusively } \\
\text { pollution exposure to } \\
\text { non-traffic related air pollution }\end{array}$ \\
\hline
\end{tabular}


The selection process of the articles was done iteratively in three stages, based on all the inclusion criteria previously defined. In the first stage, one author screened all titles and removing the titles of publications that are not relevant, and then the others authors screened the remaining publications based on abstract. Thus, after having screening the publications based on titles and abstracts, the last step will be to evaluate the eligibility of the full articles remaining.

\subsection{Data Extraction, Management and Synthesis}

For all included studies, according to the inclusion criteria on consensus of two reviewers (HL \& VA), data have been extracted and synthesized in a table. Data extracted included authors, year of publication, the location, the study design, the type of exposure variable and the different respiratory symptoms or respiratory diseases found in these studies as health outcomes.

\section{Results}

\subsection{Search Results and Flow Chart}

The initial research produced a total of 192 publications from the various databases and other sources. After removing 18 duplicates, 136 publications were excluded after an evaluation, based on a title analysis, and abstract analysis. This left a final total of 38 studies whose full text articles were reviewed for eligibility. At the end of the review process, 13 publications met the eligibility criteria and were included in the review. The PRISMA flow diagram below (Figure 1) summarizes the study selection process, including the number of publications found, the review process, and the reasons for exclusion of publications.

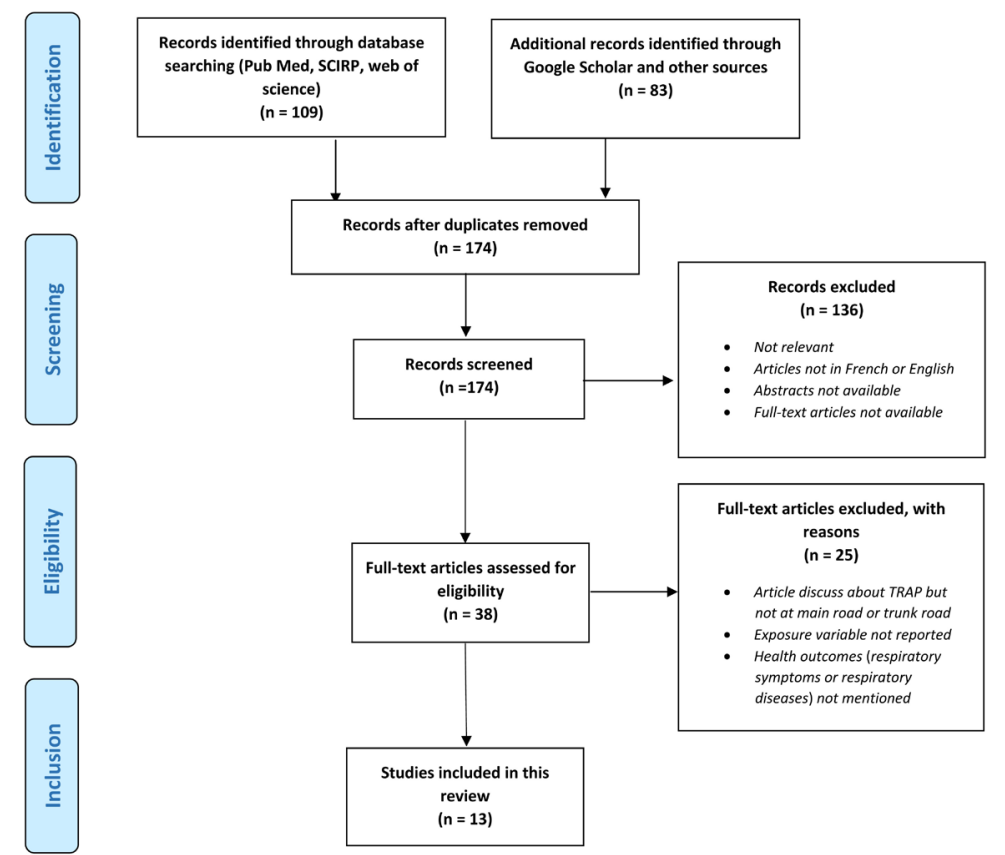

Figure 1. Preferred Reporting Item for Systematic Reviews and Meta-Analyses (PRISMA) flow diagram for article selection. 


\subsection{Study Design, Site and Population Studied}

On the 13 articles included (see Table 2), 6 were a cohort study and 6 were a cross-sectional study; one article used a case-control study and a cross-sectional study. The studies were implemented in Europe (Switzerland, UK, Netherlands, Sweden, Italy, Germany), in USA, in Asia (China, Japan) and in Oceania (Australia). Different populations were studied such as residents or children in class.

\subsection{Exposure Variables Measured in Studies Included in the Review}

Three variables were used to estimate the exposure to traffic-related air pollution: distance or proximity from a major road, traffic intensity and pollutants measured. As shown in Table 2, most of articles (11 on 13) based the characterization of the exposure to traffic-related air pollution on the distance by using different approaches. Among them were distance to closet main street per 100 $\mathrm{m}$, distance to nearest main road ( $\leq 30 \mathrm{~m} ; 60 \mathrm{~m} ; 90 \mathrm{~m} ; 120 \mathrm{~m} ; 150 \mathrm{~m}$ ) [26], distance to major road $(<200 \mathrm{~m}$ or $\geq 200 \mathrm{~m}$ ) [28], distance from trunk road ( 0 - 49 $\mathrm{m}, \geq 50 \mathrm{~m})$ [35].

Six articles used traffic intensity to characterize the exposure to traffic-related air pollution. Traffic intensity were defined by Truck traffic density, car traffic density, $<10,000$ vehicles per day versus $\geq 10,000$ vehicles per days [12], number of cars per minute $(0-1 \mathrm{cars} / \mathrm{min} ; 2-5 \mathrm{cars} / \mathrm{min} ; 6-10 \mathrm{cars} / \mathrm{min}$ and $>10$ cars/min) [31] or per 12 hours [35].

Seven articles used pollutants measure to appreciate the exposure to traffic-related air pollution. Several pollutants were measured such as $\mathrm{PM}_{10}$ [25] [30] [34]; $\mathrm{PM}_{2.5}$ [29] [30]; $\mathrm{NO}_{\mathrm{x}}$ [30] [31], $\mathrm{NO}_{2}$ [28] [29] [30] [32] [34] [35], $\mathrm{NO}$ [30], Benzene [29], $\mathrm{BC}[30]$ [32] and $\mathrm{SO}_{2}$ [35].

\subsection{Effects on Respiratory Health}

Articles included showed effects of traffic-related air pollution on respiratory health.

These effects could be mostly classified in two groups: effects found based on the distance to road (Table 3 ) and effects found based on the concentrations of pollutants (Table 4).

$\mathrm{Hu}$ et al. found that compared to participants with a home road distance of $>200 \mathrm{~m}$, participants with a home road distance of $<100 \mathrm{~m}$ or $100-200 \mathrm{~m}$ had a higher prevalence of chronic cough, with an odds ratio of 2.54 (95\% CI: 1.57 4.10 ) and 1.97 (95\% CI: 1.16 - 3.37), respectively [27]. In the same vein, Bowate et al. also found that living $<200 \mathrm{~m}$ from a major road and $\mathrm{NO}_{2}$ exposure was associated with increased current asthma and current wheeze prevalence (adjusted OR 1.49; 95\% CI: 1.09 - 2.05 and 1.61, 95\% CI: $1.19-2.19$, respectively) [28]. Regarding the results reported by Mc Connell et al., living < $75 \mathrm{~m}$ was significantly associated with a higher prevalence of asthma (adjusted OR 1.29; 95\% CI: 1.01 - 1.66), and a current wheeze (adjusted OR 1.40; 95\% CI: $1.09-1.78$ ) 
Table 2. Studies about TRAP and respiratory health on population living/working along trunk road included in the review.

\begin{tabular}{|c|c|c|c|c|c|c|c|}
\hline Author & $\begin{array}{c}\text { Year of } \\
\text { publication }\end{array}$ & $\begin{array}{l}\text { Type } \\
\text { of study }\end{array}$ & $\begin{array}{l}\text { Location } \\
\text { (city) }\end{array}$ & $\begin{array}{l}\text { Population } \\
\text { studied }\end{array}$ & $\begin{array}{l}\text { Exposure } \\
\text { variables }\end{array}$ & $\begin{array}{l}\text { Key Health } \\
\text { Outcomes }\end{array}$ & Key findings \\
\hline $\begin{array}{l}\text { Bayer- } \\
\text { Oglesby } \\
{[25]}\end{array}$ & 2006 & $\begin{array}{l}\text { Cohort } \\
\text { study }\end{array}$ & Switzerland & Adults & $\begin{array}{l}\text { - Distance } \\
\text { (distance to } \\
\text { closest main } \\
\text { street } \\
\text { (per } 100 \mathrm{~m} \text { ); } \\
\text { living within } \\
20 \text { m of a } \\
\text { main street) } \\
\text { - Pollutant } \\
\text { measured } \\
\text { - PM10 }\end{array}$ & $\begin{array}{l}\text { - RS } \\
\text { - Attack of } \\
\text { breathlessness } \\
\text { - Wheezing } \\
\text { - Regular cough } \\
\text { - Regular phlegm }\end{array}$ & $\begin{array}{l}\text { The risk of attacks of } \\
\text { breathlessness increased } \\
\text { for all subjects by } 13 \% \\
\text { per } 500 \text {-m increment in } \\
\text { the length of main street } \\
\text { segments within } 200 \mathrm{~m} \text { of } \\
\text { the home and decreased } \\
\text { in never smokers by } \\
12 \% \text { per } 100 \text { m increment } \\
\text { in distance from home } \\
\text { to a main street } \\
\text { Living within } 20 \mathrm{~m} \text { of a } \\
\text { main street increased } \\
\text { the risk of regular } \\
\text { phlegm by } 15 \% \text { and } \\
\text { wheezing with } \\
\text { breathing problems by } \\
34 \% \text { in never smokers } \\
\text { These findings among a } \\
\text { general population } \\
\text { provide strong } \\
\text { confirmation that living } \\
\text { near busy streets } \\
\text { leads to adverse } \\
\text { respiratory health effects }\end{array}$ \\
\hline $\begin{array}{l}\text { Venn } \\
\text { et al. } \\
{[26]}\end{array}$ & 2001 & $\begin{array}{l}\text { Case } \\
\text { control/ } \\
\text { Cross } \\
\text { sectional }\end{array}$ & $\begin{array}{c}\text { UK } \\
\text { (Nottingham) }\end{array}$ & $\begin{array}{c}\text { Children } \\
\text { (4 to } 11 \text { years) } \\
\text { and adolescents } \\
\text { (11 to } 16 \text { years) }\end{array}$ & $\begin{array}{l}\text { Distance to } \\
\text { nearest } \\
\text { main road } \\
(\leq 30 \mathrm{~m} ; \\
60 \mathrm{~m} ; 90 \mathrm{~m} ; \\
120 \mathrm{~m} ; \\
150 \mathrm{~m})\end{array}$ & $\begin{array}{l}\text { - RS } \\
\text { - Wheezing } \\
\text { - RD } \\
\text { - Asthma }\end{array}$ & $\begin{array}{l}\text { - Among children living } \\
\text { within } 150 \mathrm{~m} \text { of a main } \\
\text { road, the risk of wheeze } \\
\text { increased. And most of } \\
\text { the increased risk was } \\
\text { localized to within } 90 \mathrm{~m} \\
\text { of the roadside. } \\
\text { - Living within } \\
\text { approximately } 90 \mathrm{~m} \text { of a } \\
\text { main road is associated } \\
\text { with a proximity-related } \\
\text { increase in the risk of } \\
\text { wheezing illness in } \\
\text { children }\end{array}$ \\
\hline $\begin{array}{c}\text { Hu et al. } \\
\text { [27] }\end{array}$ & 2016 & $\begin{array}{l}\text { Cohort } \\
\text { study }\end{array}$ & $\begin{array}{l}\text { China } \\
\text { (Beijing) }\end{array}$ & $\begin{array}{l}\text { Adults } \\
(>35 \text { years) } \\
\text { long-term } \\
\text { residents } \\
\text { ( } \geq 3 \text { years) }\end{array}$ & $\begin{array}{l}\text { Distance } \\
(<100 \mathrm{~m} \text {; } \\
100-200 \mathrm{~m} \\
\text { or }>200 \mathrm{~m})\end{array}$ & $\begin{array}{l}\text { - RS } \\
\text { - Chronic cough } \\
\text { - Shortness } \\
\text { of breath }\end{array}$ & $\begin{array}{l}\text { Participants living a } \\
\text { shorter distance from } \\
\text { major roads had lower } \\
\text { percentage of predicted } \\
\text { FEV in } 1 \mathrm{~s} \text {. } \\
\text { - Long term exposure to } \\
\text { traffic related air } \\
\text { pollution in people } \\
\text { living near major roads } \\
\text { in Beijing is associated } \\
\text { with lower lung function, } \\
\text { airway acidification, and } \\
\text { a higher prevalence } \\
\text { of chronic cough }\end{array}$ \\
\hline
\end{tabular}


Bowate

et al.

[28]

2017 Longitudina
study

(data from

established

cohort study)
Australia
(Tasmania)

Janssen

et al.

[29]
Crosssectional study
2003
Garshick

et al.

[12]
Netherlands

Children in

classes 4 - 8 (7 - 12 years old)
- Distance to

major road

$(<200 \mathrm{~m}$;

$\geq 200 \mathrm{~m}$ )

(45 - 50 years)

- Pollutant

measured

- $\mathrm{NO}_{2}$
- RS

- Wheeze

- RD

- Asthma
- Living < $200 \mathrm{~m}$ from a major road was associated with increased prevalence of current asthma and wheeze, and lower lung function

- Over the 5-year period, higher $\mathrm{NO}_{2}$ exposures were associated with increased asthma prevalence
- Traffic

intensity

- Truck traffic density $\quad$ RS

(vehicles/ - Wheeze

weekday) - Asthma ever

- Car traffic - Current density conjunctivitis (vehicles/ - Hay fever ever weekday) - Current itchy

- Distance rash

- Distance - Eczema ever

schools - Current phlegm

motorways

(m)

- Distance

home -

motorway (m)

- Pollutants

measured

- $\mathrm{PM}_{2.5}$

- $\mathrm{NO}_{2}$

- Benzene
- Air pollutants that increased near motorways with high truck traffic counts

- Respiratory symptoms were increased also near motorways with high truck traffic counts.
- Men living within $50 \mathrm{~m}$ of a major roadway were more likely to report persistent wheeze compared with those living more than $400 \mathrm{~m}$ away

- The risk was observed only for those living within $50 \mathrm{~m}$ of heavily trafficked road ( $\geq 10,000$ vehicles/24h)

- Exposure to vehicular emissions by living near busy roadways contribute to symptoms of chronic respiratory

diseases in adults 


\section{Continued}

\begin{tabular}{|c|c|c|c|c|c|c|c|}
\hline $\begin{array}{l}\text { Kim } \\
\text { et al. } \\
{[30]}\end{array}$ & 2004 & $\begin{array}{l}\text { Cross- } \\
\text { sectional } \\
\text { study }\end{array}$ & $\begin{array}{c}\text { US } \\
\text { (San Francisco) }\end{array}$ & Children & $\begin{array}{l}\text { - Traffic } \\
\text { intensity } \\
(90,000- \\
210,000 \\
\text { veh/day) } \\
\text { - Distance } \\
\text { (<300 m } \\
\text { downwind) } \\
\text { - Pollutants } \\
\text { measured } \\
-\mathrm{PM}_{10} \\
-\mathrm{PM}_{2.5} \\
-\mathrm{BC} \\
-\mathrm{NO}_{\mathrm{x}} \\
-\mathrm{NO}_{2} \\
-\mathrm{NO}^{-}\end{array}$ & $\begin{array}{l}- \text { RD } \\
\text { - Asthma } \\
\text { - Bronchitis }\end{array}$ & $\begin{array}{l}\text { - Observation of difference } \\
\text { concentrations between } \\
\text { schools nearby versus } \\
\text { more distant (or upwind) } \\
\text { from major roads } \\
\text { - Association between } \\
\text { respiratory symptoms } \\
\text { and traffic related } \\
\text { pollutants } \\
\text { - Findings support the } \\
\text { hypothesis that TRAP } \\
\text { is associated with } \\
\text { respiratory symptoms }\end{array}$ \\
\hline $\begin{array}{l}\text { Lindgren } \\
\text { et al. } \\
{[31]}\end{array}$ & 2009 & $\begin{array}{l}\text { Cross- } \\
\text { sectional } \\
\text { study }\end{array}$ & $\begin{array}{l}\text { Southern } \\
\text { Sweden }\end{array}$ & $\begin{array}{c}\text { Adults } \\
(18-77 \text { years })\end{array}$ & $\begin{array}{l}\text { - Traffic } \\
\text { intensity } \\
(0-1 \\
\text { cars/min; } \\
2-5 \text { cars/min; } \\
6-10 \\
\text { cars/min } \\
\text { and }>10 \\
\text { cars/min } \\
\text { based upon } \\
24 \text {-hour } \\
\text { mean levels }) \\
\text { - Pollutants } \\
\text { measured } \\
\text { - NO } \\
\text { modeled } \\
\text { exposure })\end{array}$ & $\begin{array}{l} \\
\text { - RS } \\
\text { - Asthma } \\
\text { symptoms } \\
\text { - Chronic } \\
\text { bronchitis } \\
\text { symptoms } \\
\text { - RD } \\
\text { - Asthma } \\
\text { - COPD } \\
\text { - CBE } \\
\end{array}$ & $\begin{array}{l}\text { - Living close to traffic } \\
\text { (within } 100 \mathrm{~m} \text { of a road } \\
\text { with }>10 \text { cars/minute) } \\
\text { was associated with } \\
\text { prevalence of asthma } \\
\text { diagnosis and COPD } \\
\text { diagnosis, and symptoms } \\
\text { of asthma and bronchitis. } \\
\text { - Annual average } \mathrm{NO}_{\mathrm{x}} \\
\text { was associated with } \\
\text { COPD diagnosis and } \\
\text { symptoms of asthma } \\
\text { and chronic bronchitis }\end{array}$ \\
\hline $\begin{array}{l}\text { Hoek } \\
\text { et al. } \\
{[32]}\end{array}$ & 2002 & $\begin{array}{l}\text { Cohort } \\
\text { study }\end{array}$ & Netherlands & $\begin{array}{c}\text { Adults } \\
(55-69 \text { years })\end{array}$ & $\begin{array}{l}\text { - Distance } \\
\text { - Pollutants } \\
\text { measured } \\
\text { - } \mathrm{BC} \\
-\mathrm{NO}_{2}\end{array}$ & $\begin{array}{l}\text { - RD } \\
\text { - Lung cancer } \\
\text { - Cardio- } \\
\text { pulmonary } \\
\text { diseases }\end{array}$ & $\begin{array}{l}\text { - Cardiopulmonary } \\
\text { mortality was } \\
\text { associated with living } \\
\text { near a major road. } \\
\text { - Long-term exposure } \\
\text { to traffic-related air } \\
\text { pollution may } \\
\text { shorten life expectancy }\end{array}$ \\
\hline $\begin{array}{c}\text { Migliore } \\
\text { et al. } \\
{[33]}\end{array}$ & 2009 & $\begin{array}{l}\text { Cross- } \\
\text { sectional } \\
\text { study }\end{array}$ & Italy & $\begin{array}{c}\text { Children } \\
(6-7 \text { years }) \\
\text { and adolescents } \\
\text { (13 - } 14 \text { years })\end{array}$ & $\begin{array}{l}\text { - Traffic } \\
\text { intensity }\end{array}$ & $\begin{array}{l}\text { - RS } \\
\text { - Asthma } \\
\text { symptoms } \\
\text { - Cough or } \\
\text { phlegm }\end{array}$ & $\begin{array}{l}\text { Traffic density was } \\
\text { weakly associated } \\
\text { with asthma } \\
\text { symptoms but there } \\
\text { was a stronger } \\
\text { association with } \\
\text { cough or phlegm. } \\
\text { - Children living in } \\
\text { zones with intense } \\
\text { traffic are at higher } \\
\text { risk for respiratory } \\
\text { effects. }\end{array}$ \\
\hline
\end{tabular}




\begin{tabular}{|c|c|c|c|c|c|c|c|}
\hline $\begin{array}{l}\text { Schikowski } \\
\qquad \text { et al. } \\
{[34]}\end{array}$ & 2005 & $\begin{array}{c}\text { Cross- } \\
\text { sectional } \\
\text { study }\end{array}$ & Germany & $\begin{array}{c}\text { Adults } \\
\text { (Women) }\end{array}$ & $\begin{array}{l}\text { - Distance } \\
(<100 \mathrm{~m}, \\
\geq 100 \mathrm{~m}) \\
\text { - Pollutants } \\
\text { measured } \\
-\mathrm{NO}_{2} \\
-\mathrm{PM}_{10}\end{array}$ & $\begin{array}{l}\text { - RS } \\
\text { - Chronic cough } \\
\text { with phlegm } \\
\text { - Frequent cough } \\
\text { - RD } \\
\text { - COPD } \\
\text { - Chronic } \\
\text { bronchitis }\end{array}$ & $\begin{array}{l}\text { - COPD and pulmonary } \\
\text { function were strongest } \\
\text { affected by PM10 a } \\
\text { traffic related exposure. } \\
\text { - Women living less than } \\
100 \mathrm{~m} \text { from a busy road } \\
\text { also had a significantly } \\
\text { decreased lung function } \\
\text { and COPD was } 1.79 \\
\text { times more likely than } \\
\text { for those living } \\
\text { farther away } \\
\text { - Chronic exposure to } \\
\text { PM }{ }_{10}, \mathrm{NO}_{2} \text { and living } \\
\text { near a major road might } \\
\text { increase the risk of } \\
\text { developing COPD and } \\
\text { can have a detrimental } \\
\text { effect on lung function }\end{array}$ \\
\hline $\begin{array}{c}\text { Shima } \\
\text { et al. } \\
{[35]}\end{array}$ & 2003 & $\begin{array}{l}\text { Cohort } \\
\text { study }\end{array}$ & Japan & $\begin{array}{l}\text { Children } \\
\text { ( } 6-9 \text { years) }\end{array}$ & $\begin{array}{l}\text { - Traffic } \\
\text { intensity } \\
\text { (number of } \\
\text { vehicles/12 } \\
\text { hours) } \\
\text { - Distance } \\
\text { from } \\
\text { trunk road } \\
(0-49 \mathrm{~m} \text {; } \\
\geq 50 \mathrm{~m} \text {; } \\
\text { rural area) } \\
\text { - Pollutants } \\
\text { measured } \\
\text { - } \mathrm{NO}_{2} \\
-\mathrm{SPM}^{-} \\
-\mathrm{SO}_{2}\end{array}$ & $\begin{array}{l}\text { - RS } \\
\text { - Bronchitis ever } \\
\text { - Chronic cough } \\
\text { - Chronic phlegm } \\
\text { - Wheeze } \\
\text { - RD } \\
\text { - Asthma }\end{array}$ & $\begin{array}{l}\text { - The prevalence of } \\
\text { asthma increased with } \\
\text { increases of air pollution. } \\
\text { - Findings suggest that } \\
\text { traffic-related air } \\
\text { pollution may be of a } \\
\text { particular importance } \\
\text { in the development of } \\
\text { asthma among children } \\
\text { living near major trunk } \\
\text { roads with heavy traffic }\end{array}$ \\
\hline $\begin{array}{c}\text { McConnel } \\
\text { et al. } \\
{[36]}\end{array}$ & 2006 & $\begin{array}{l}\text { Cohort } \\
\text { study }\end{array}$ & $\begin{array}{l}\text { US (Southern } \\
\text { California) }\end{array}$ & $\begin{array}{l}\text { Children } \\
\text { (5- } 7 \text { years) }\end{array}$ & $\begin{array}{l}\text { Distance } \\
(<75 \mathrm{~m} ; \\
75-150 \mathrm{~m} ; \\
150 \mathrm{~m}- \\
300 \mathrm{~m} ; \\
>300 \mathrm{~m})\end{array}$ & $\begin{array}{l}\text { - RS } \\
\text { - Current wheeze } \\
\text { - RD } \\
\text { - Asthma }\end{array}$ & $\begin{array}{l}\text { Residence within } 75 \mathrm{~m} \\
\text { of a major road was } \\
\text { associated with an } \\
\text { increased risk of } \\
\text { lifetime asthma }\end{array}$ \\
\hline
\end{tabular}

Definition of abbreviations. RS: Respiratory symptoms; RD: Respiratory diseases; BC: black carbon; NO: nitric oxide; $\mathrm{NO}_{x}$ : total nitrogen oxides; $\mathrm{NO}_{2}$ : nitrogen dioxide; $\mathrm{PM}_{2.5}$ : particulate matter of aerodynamic diameter $2.5 \mu \mathrm{m}$ or less; $\mathrm{PM}_{10}$ : particulate matter of aerodynamic diameter $10 \mu \mathrm{m}$ or less. COPD: Chronic Obstructive Pulmonary Disease; CBE: Chronic Bronchitis Emphysema; FEV: Forced expiratory volume.

[36]. As for Schikowski et al., living < $100 \mathrm{~m}$ from major road was significantly associated with an increased risk of frequent cough (adjusted OR 1.24; 95\% CI: 1.03 - 1.49) and COPD (adjusted OR 1.79; 95\% CI: 1.06 - 3.02) [34].

Based on pollutants concentrations, several authors found associations between fine particles or nitrogen oxide and respiratory symptoms or diseases. Kim et al. found that exposure to NO was significantly associated to development of bronchitis (adjusted OR 1.79; 95\% CI: 1.06 - 3.02) [30]. Schikowski et al. also found association between exposure to $\mathrm{NO}_{2}$ and chronic bronchitis 
Table 3. Some significant associations between respiratory symptoms or diseases and distance to major road.

\begin{tabular}{|c|c|c|c|}
\hline Authors & $\mathrm{RS} / \mathrm{RD}$ & Exposure (Distance) & OR $(95 \% \mathrm{CI})$ \\
\hline \multirow[t]{5}{*}{ Hu et al. } & Chronic cough & & \\
\hline & & $<100 \mathrm{~m}$ & $2.54(1.57-4.10)$ \\
\hline & & $100-200 \mathrm{~m}$ & $1.97(1.16-3.37)$ \\
\hline & & $>200 \mathrm{~m}$ & 1 \\
\hline & Current asthma & & \\
\hline \multirow[t]{8}{*}{ Bowate et al. } & & Living $<200 \mathrm{~m}$ from a major road & $1.49(1.09-2.05)$ \\
\hline & Current wheeze & & \\
\hline & & Living $<200 \mathrm{~m}$ from a major road & $1.61(1.19-2.19)$ \\
\hline & Lifetime asthma & & \\
\hline & & $>300$ & 1 \\
\hline & & $150-300$ & $0.92(0.73-1.15)$ \\
\hline & & $75-150$ & $1.06(0.82-1.36)$ \\
\hline & & $<75$ & $1.29(1.01-1.66)$ \\
\hline
\end{tabular}

Prevalent asthma

\begin{tabular}{|c|c|c|c|}
\hline & & $>300$ & 1 \\
\hline \multirow[t]{10}{*}{ Mc Connell et al. } & & $150-300$ & $1.04(0.82-1.33)$ \\
\hline & & $75-150$ & $1.33(1.02-1.72)$ \\
\hline & & $<75$ & $1.50(1.16-1.95)$ \\
\hline & Current wheeze & & \\
\hline & & $>300$ & 1 \\
\hline & & $150-300$ & $1.02(0.82-1.27)$ \\
\hline & & $75-150$ & $1.30(1.02-1.66)$ \\
\hline & & $<75$ & $1.40(1.09-1.78)$ \\
\hline & Frequent cough & & \\
\hline & & $\begin{array}{c}<100 \mathrm{~m} \text { from major road wi } \\
10,000 \text { cars/day compared to }>100 \mathrm{~m}\end{array}$ & $1.24(1.03-1.49)$ \\
\hline \multirow[t]{2}{*}{ Schikowski et al. } & $C O P D$ & & \\
\hline & & $\begin{array}{c}<100 \mathrm{~m} \text { from major road wi } \\
10,000 \text { cars/day compared to }>100 \mathrm{~m}\end{array}$ & $1.79(1.06-3.02)$ \\
\hline
\end{tabular}

RS: Respiratory symptoms; RD: Respiratory diseases; OR: Odd ratio; COPD: Chronic Obstructive Pulmonary Disease.

(adjusted OR 1.25; 95\% CI: 1.00 - 1.58), frequent cough (adjusted OR 1.13; 95\% CI: 1.01 - 1.27) and COPD (adjusted OR 1.39; 95\% CI: 1.20 - 1.63). Another result found by Schikowski et al. is the association between $\mathrm{PM}_{10}$ and COPD. $\mathrm{He}$ found that an increase of $7 \mathrm{of} \mu \mathrm{g} / \mathrm{m}^{3} \mathrm{PM} 10$ was associated with an increased risk of COPD (adjusted OR 1.33; 95\% CI: 1.03 - 1.72) [34]. 
Table 4. Some significant associations between respiratory symptoms or diseases and air pollution concentrations.

\begin{tabular}{|c|c|c|c|}
\hline Authors & RS/RD & Exposure (Pollutants) & OR $(95 \% \mathrm{CI})$ \\
\hline \multirow{3}{*}{ Kim et al. } & Bronchitis & & \\
\hline & & $\mathrm{NO}_{\mathrm{x}}$ & $1.05(1.01,1.08)$ \\
\hline & & NO & $1.05(1.02,1.09)$ \\
\hline \multirow{7}{*}{ Schikowski et al. } & $\begin{array}{c}\text { Chronic bronchitis by } \\
\text { physician diagnosis }\end{array}$ & & \\
\hline & & $\mathrm{NO}_{2}\left[16 \mu \mathrm{g} / \mathrm{m}^{3}\right]$ & $1.25(1.00-1.58)$ \\
\hline & Frequent cough & & \\
\hline & & $\mathrm{NO}_{2}\left[16 \mu \mathrm{g} / \mathrm{m}^{3}\right]$ & $1.13(1.01-1.27)$ \\
\hline & $C O P D$ & & \\
\hline & & $\mathrm{NO}_{2}\left[16 \mu \mathrm{g} / \mathrm{m}^{3}\right]$ & $1.39(1.20-1.63)$ \\
\hline & & $\mathrm{PM}_{10}\left[7 \mu \mathrm{g} / \mathrm{m}^{3}\right]$ & $1.33(1.03-1.72)$ \\
\hline \multirow{2}{*}{ Janseens et al. } & Hay fever ever & & \\
\hline & & $\mathrm{PM}_{2.5}$ & $2.28(1.13-4.57)$ \\
\hline
\end{tabular}

RS: Respiratory symptoms; RD: Respiratory diseases; OR: Odd ratio; COPD: Chronic Obstructive Pulmonary Disease.

In the rest of this paragraph, we presented some results that combined the effects of distance to major road, the concentrations of pollutants or the intensity of traffic on respiratory health. Bowate et al. also found that living $<200 \mathrm{~m}$ from a major road and $\mathrm{NO}_{2}$ exposure was associated with increased current asthma prevalence (adjusted OR 1.49; 95\% CI: 1.09 - 2.05 and aOR 1.10, 95\% CI: 0.96 1.27 , respectively) [28]. In the same vein, Garshick et al. reported that exposure to vehicular emissions by living near busy roadways contribute to symptoms of chronic respiratory diseases in adults [12] and $\mathrm{Hu}$ et al. reported that long-term exposure to traffic-related air pollution in people living near major roads in Beijing is associated with lower lung function, airway acidification and a higher prevalence of chronic cough [27]. Lindgren et al. found that living close to traffic (within $100 \mathrm{~m}$ of a road with $>10$ cars/minute) and annual average $\mathrm{NO}_{\mathrm{x}}$ was associated with prevalence of asthma diagnosis and COPD diagnosis and symptoms of asthma and bronchitis [31]. Concerning the children's, Migliore et al. found that children living in zones with intense traffic are at higher risk for respiratory effects [33] and Kim et al. also reported an observation of a difference concentrations between schools nearby versus more distant (or upwind) from major roads and association between respiratory symptoms and traffic related pollutants [30]. Shima et al., findings confirmed that traffic-related air pollution may be of a particular importance in the development of asthma among children living near major trunk roads with heavy traffic [35]. Hoek et al. goes much deeper by establishing that long-term exposure to traffic-related air pollution may shorten life expectancy due to fact that cardiopulmonary mortality was associated with living near a major road [32]. 
The different effects reported in this review can be classified in respiratory symptoms and respiratory diseases. The main respiratory symptoms found were wheezing [12] [25] [26] [28] [29] [36], cough [12] [25] [27] [33] [34] [35]. As respiratory diseases, the articles mentioned asthma [26] [28] [30] [31] [35] [36], bronchitis ([30] [31] [34], chronic respiratory diseases [12] [31] [34].

\section{Discussion}

This systematic review focused on different methods to estimate the exposure to traffic-related air pollution and its consequences on respiratory health in population working or living along or near the trunk road. So, what we were going to discuss in this paper was not the degree of association between exposure to traffic-related and respiratory health but we would like to highlight the different approaches used for these relationships.

The authors found only 13 studies that meet the criteria and no study in Africa. To characterize the exposure to traffic-related air pollution, studies used distance to road [12] [25]-[30] [32] [34] [35] [36], traffic density [12] [26] [29] [30] [31] [33] [35] and pollutants measure [25] [28] [29] [30] [31] [32] [34] [35]. Several previous systematic reviews came to the same conclusion in their study [20] [37] [38].

Based on these three characterization approaches, the authors of different papers have estimated the health outcomes.

The main health outcomes found were wheeze and cough for respiratory symptoms, asthma and bronchitis for respiratory diseases. This is consistent with some results in literature. Indeed Weinmayr et al., found that cough was one of key health respiratory symptom outcome [39]. Sylla et al. and Weinmayr et al. found in their review that asthma was one of key health respiratory disease outcome [20] [39].

Whether to characterize exposure to air pollution related to road traffic or to determine the effects of this exposure on respiratory health, the different articles included did not specifically address the particularity of roundabouts. Certainly, because these studies did not take place in the context of major West African cities. In fact, in these cities, urbanization is not always well carried out and the populations are enthusiastic to settle along the roads but also around the roundabouts with heavy traffic for their commercial activities [40] [41]. But living or working closet to high-traffic may have an additional risk depending on the location in relation to roundabouts and this situation has not been considered in different studies included.

\section{Conclusion}

In the present study, the authors found that distance to road, traffic density and pollutants measure are the usual methods to characterize the exposure to traffic-related air and his consequences on respiratory health. No study mentioned the situation about specifically populations around the roundabouts. But the 
situation in Africa's cities is different regarding the urbanization and the enthusiasm of population to settle along high traffic road and roundabouts. The authors suggest that it would be very useful if research focused on the populations living around the roundabouts in order to determine their possible overexposure to air pollution linked to traffic.

\section{Acknowledgements}

Le financement de cette étude a été accordé par le Centre de Recherches pour le Développement International (CRDI) dans le cadre du projet "Chairepol" (projet CRDI 107347) de la Communauté de pratique en écosanté pour l'Afrique de l'Ouest et du Centre (CoPES-AOC)/Chaire ECOSANTE.

\section{Conflicts of Interest}

The authors declare no conflicts of interest regarding the publication of this paper.

\section{References}

[1] Landrigan, P.J., Fuller, R., Acosta, N.J.R., Adeyi, O., Arnold, R., Basu, N.N., et al. (2018) The Lancet Commission on Pollution and Health. Lancet, 391, 462-512. https://doi.org/10.1016/S0140-6736(17)32345-0

[2] Blazhevska, V. (2016) Vast Majority of World-6.76 Billion People-Living with Excessive Air Pollution-UN Report. United Nations Sustainable Development, New York.

https://www.un.org/sustainabledevelopment/blog/2016/09/vast-majority-of-world6-76-billion-people-living-with-excessive-air-pollution-un-report/

[3] World Health Organization (2018) Ambient (Outdoor) Air Pollution. https://www.who.int/news-room/fact-sheets/detail/ambient-(outdoor)-air-quality-a nd-health

[4] Guarnieri, M. and Balmes, J.R. (2014) Outdoor Air Pollution and Asthma. Lancet, 383, 1581-1592. https://doi.org/10.1016/S0140-6736(14)60617-6

[5] Brauer, M., Amann, M., Burnett, R.T., Cohen, A., Dentener, F., Ezzati, M., et al. (2012) Exposure Assessment for Estimation of the Global Burden of Disease Attributable to Outdoor Air Pollution. Environmental Science \& Technology, 46, 652-660. https://doi.org/10.1021/es2025752

[6] Perez, L., Declercq, C., Iñiguez, C., Aguilera, I., Badaloni, C., Ballester, F., et al. (2013) Chronic Burden of Near-Roadway Traffic Pollution in 10 European Cities (APHEKOM Network). European Respiratory Journal, 42, 594-605. https://doi.org/10.1183/09031936.00031112

[7] Krzyżanowski, M., Kuna-Dibbert, B. and Schneider, J. (2005) Health Effects of Transport-Related Air Pollution. World Health Organization Europe, Copenhagen, $190 \mathrm{p}$.

[8] Hegseth, M.N., Oftedal, B.M., Höper, A.C., Aminoff, A.L., Thomassen, M.R., et al. (2019) Self-Reported Traffic-Related Air Pollution and Respiratory Symptoms among Adults in an Area with Modest Levels of Traffic. PLOS ONE, 14, e0226221. https://doi.org/10.1371/journal.pone.0226221

[9] Djossou, J., Léon, J.-F., Akpo, A.B., Liousse, C., Yoboué, V., Bedou, M., et al. (2018) Mass Concentration, Optical Depth and Carbon Composition of Particulate Matter 
in the Major Southern West African Cities of Cotonou (Benin) and Abidjan (Côte d'Ivoire). Atmospheric Chemistry and Physics, 18, 6275-6291. https://doi.org/10.5194/acp-18-6275-2018

[10] Singh, A., Avis, W.R. and Pope, F.D. (2020) Visibility as a Proxy for Air Quality in East Africa. Environmental Research Letters, 15, Article ID: 084002. https://doi.org/10.1088/1748-9326/ab8b12

[11] Liousse, C. and Galy-Lacaux, C. (2010) Urban Pollution in West Africa. Météorologie , 8, 45-49.

[12] Garshick, E., Laden, F., Hart, J.E. and Caron, A. (2003) Residence Near a Major Road and Respiratory Symptoms in U.S. Veterans. Epidemiology, 14, 728-736. https://doi.org/10.1097/01.ede.0000082045.50073.66

[13] Kan, H., Heiss, G., Rose, K.M., Whitsel, E., Lurmann, F. and London, S.J. (2007) Traffic Exposure and Lung Function in Adults: The Atherosclerosis Risk in Communities Study. Thorax, 62, 873-879. https://doi.org/10.1136/thx.2006.073015

[14] Nitta, H., Sato, T., Nakai, S., Maeda, K., Aoki, S. and Ono, M. (1993) Respiratory Health Associated with Exposure to Automobile Exhaust. I. Results of Cross-Sectional Studies in 1979, 1982, and 1983. Archives of Environmental Health: An International Journal, 48, 53-58. https://doi.org/10.1080/00039896.1993.9938393

[15] Brunekreef, B., Stewart, A.W., Anderson, H.R., Lai, C.K.W., Strachan, D.P., Pearce, N., et al. (2009) Self-Reported Truck Traffic on the Street of Residence and Symptoms of Asthma and Allergic Disease: A Global Relationship in ISAAC Phase 3. Environmental Health Perspectives, 117, 1791-1798. https://doi.org/10.1289/ehp.0800467

[16] Cook, A.G., deVos, A.J., Pereira, G., Jardine, A. and Weinstein, P. (2011) Use of a Total Traffic Count Metric to Investigate the Impact of Roadways on Asthma Severity: A Case-Control Study. Environmental Health, 10, Article No. 52. https://doi.org/10.1186/1476-069X-10-52

[17] Coker, E. and Kizito, S. (2018) A Narrative Review on the Human Health Effects of Ambient Air Pollution in Sub-Saharan Africa: An Urgent Need for Health Effects Studies. International Journal of Environmental Research and Public Health, 15, 427. https://doi.org/10.20944/preprints201801.0058.v1

[18] Matz, C.J., Egyed, M., Hocking, R., Seenundun, S., Charman, N. and Edmonds, N. (2019) Human Health Effects of Traffic-Related Air Pollution (TRAP): A Scoping Review Protocol. Systematic Reviews, 8, Article No. 223.

https://doi.org/10.1186/s13643-019-1106-5

[19] Madureira, J., Brancher, E.A., Costa, C., Aurino de Pinho, R. and Teixeira, J.P. (2019) Cardio-Respiratory Health Effects of Exposure to Traffic-Related Air Pollutants While Exercising Outdoors: A Systematic Review. Environmental Research, 178, Article ID: 108647. https://doi.org/10.1016/j.envres.2019.108647

[20] Sylla, F.K., Faye, A., Fall, M. and Tal-Dia, A. (2017) Air Pollution Related to Traffic and Chronic Respiratory Diseases (Asthma and COPD) in Africa. Health, 9, 1378-1389. https://doi.org/10.4236/health.2017.910101

[21] Tétreault, L.-F., Perron, S. and Smargiassi, A. (2013) Cardiovascular Health, Traffic-Related Air Pollution and Noise: Are Associations Mutually Confounded? A Systematic Review. International Journal of Public Health, 58, 649-666. https://doi.org/10.1007/s00038-013-0489-7

[22] Clark, D.P.Q., Son, D.B., Bowatte, G., Senaratna, C.V., Lodge, C., Perret, J.L., et al. (2020) The Association between Traffic-Related Air Pollution and Obstructive Sleep Apnea: A Systematic Review. Sleep Medicine Reviews, 54, Article ID: 101360. 
https://doi.org/10.1016/j.smrv.2020.101360

[23] Lawin, H., Ayi Fanou, L, Hinson, A.V., Stolbrink, M., Houngbegnon, P., Kedote, N.M., et al. (2018) Health Risks Associated with Occupational Exposure to Ambient Air Pollution in Commercial Drivers: A Systematic Review. International Journal of Environmental Research and Public Health, 15, 2039.

https://doi.org/10.20944/preprints201808.0215.v1

[24] Moher, D., Liberati, A., Tetzlaff, J., Altman, D.G. and PRISMA Group (2009) Preferred Reporting Items for Systematic Reviews and Meta-Analyses: The PRISMA Statement. BMJ, 339, b2535. https://doi.org/10.1136/bmj.b2535

[25] Bayer-Oglesby, L., Schindler, C., Hazenkamp-von Arx, M.E., Braun-Fahrländer, C., Keidel, D., Rapp, R., et al. (2006) Living Near Main Streets and Respiratory Symptoms in Adults: The Swiss Cohort Study on Air Pollution and Lung Diseases in Adults. American Journal of Epidemiology, 164, 1190-1198.

https://doi.org/10.1093/aje/kwj338

[26] Venn, A.J., Lewis, S.A., Cooper, M., Hubbard, R. and Britton, J. (2001) Living Near a Main Road and the Risk of Wheezing Illness in Children. American Journal of Respiratory and Critical Care Medicine, 164, 2177-21780.

https://doi.org/10.1164/ajrccm.164.12.2106126

[27] Hu, Z.-W., Zhao, Y.-N., Cheng, Y., Guo, C.-Y., Wang, X., Li, N., et al. (2016) Living Near a Major Road in Beijing: Association with Lower Lung Function, Airway Acidification, and Chronic Cough. Chinese Medical Journal, 129, 2184-2190. https://doi.org/10.4103/0366-6999.189923

[28] Bowatte, G., Erbas, B., Lodge, C.J., Knibbs, L.D., Gurrin, L.C., Marks, G.B., et al. (2017) Traffic-Related Air Pollution Exposure over a 5-Year Period Is Associated with Increased Risk of Asthma and Poor Lung Function in Middle Age. European Respiratory Journal, 50, Article ID: 1602357. https://doi.org/10.1183/13993003.02357-2016

[29] Janssen, N.A.H., Brunekreef, B., van Vliet, P., Aarts, F., Meliefste, K., Harssema, H., et al. (2003) The Relationship between Air Pollution from Heavy Traffic and Allergic sensiTization, Bronchial Hyperresponsiveness, and Respiratory Symptoms in Dutch Schoolchildren. Environmental Health Perspectives, 111, 1512-1518. https://doi.org/10.1289/ehp.6243

[30] Kim, J.J., Smorodinsky, S., Lipsett, M., Singer, B.C., Hodgson, A.T. and Ostro, B. (2004) Traffic-Related Air Pollution Near Busy Roads: The East Bay Children's Respiratory Health Study. American Journal of Respiratory and Critical Care Medicine, 170, 520-526. https://doi.org/10.1164/rccm.200403-2810C

[31] Lindgren, A., Stroh, E., et al.(2009) Traffic-Related Air Pollution Associated with Prevalence of Asthma and COPD/Chronic Bronchitis. A Cross-Sectional Study in Southern Sweden. International Journal of Health Geographics, 8, Article No. 2. https://doi.org/10.1186/1476-072X-8-2

[32] Hoek, G., Brunekreef, B., Goldbohm, S., Fischer, P. and van den Brandt, P.A. (2002) Association between Mortality and Indicators of Traffic-Related Air Pollution in the Netherlands: A Cohort Study. Lancet, 360, 1203-1209. https://doi.org/10.1016/S0140-6736(02)11280-3

[33] Migliore, E., Berti, G., Galassi, C., Pearce, N., Forastiere, F., Calabrese, R., et al. (2009) Respiratory Symptoms in Children Living Near Busy Roads and Their Relationship to Vehicular Traffic: Results of an Italian Multicenter Study (SIDRIA 2). Environmental Health, 8, Article No. 27. https://doi.org/10.1186/1476-069X-8-27

[34] Schikowski, T., Sugiri, D., Ranft, U., Gehring, U., Heinrich, J., Wichmann, H.-E., et 
al. (2005) Long-term Air Pollution Exposure and Living Close to Busy Roads Are Associated with COPD in Women. Respiratory Research, 6, Article No. 152. https://doi.org/10.1186/1465-9921-6-152

[35] Shima, M., Nitta, Y. and Adachi, M. (2003) Traffic-Related Air Pollution and Respiratory Symptoms in Children Living along Trunk Roads in Chiba Prefecture. Japan. Journal of Epidemiology, 13, 108-119. https://doi.org/10.2188/jea.13.108

[36] McConnell, R., Berhane, K., Yao, L., Jerrett, M., Lurmann, F., Gilliland, F., Künzli, N., et al. (2006) Traffic, Susceptibility, and Childhood Asthma. Environmental Health Perspectives, 114, 766-772. https://doi.org/10.1289/ehp.8594

[37] Hamra, G.B., Laden, F., Cohen, A.J., Raaschou-Nielsen, O., Brauer, M. and Loomis, D. (2015) Lung Cancer and Exposure to Nitrogen Dioxide and Traffic: A Systematic Review and Meta-Analysis. Environmental Health Perspectives, 123, 1107-1112. https://doi.org/10.1289/ehp.1408882

[38] Brugge, D., Durant, J.L. and Rioux, C. (2007) Near-Highway Pollutants in Motor Vehicle Exhaust: A Review of Epidemiologic Evidence of Cardiac and Pulmonary Health Risks. Environmental Health, 6, Article No. 23.

https://doi.org/10.1186/1476-069X-6-23

[39] Weinmayr, G., Romeo, E., De Sario, M., Weiland, SK. and Forastiere, F. (2010) Short-Term Effects of PM10 and $\mathrm{NO}_{2}$ on Respiratory Health among Children with Asthma or Asthma-Like Symptoms: A Systematic Review and Meta-Analysis. Environmental Health Perspectives, 118, 449-457. https://doi.org/10.1289/ehp.0900844

[40] Nyassogbo, G.K. (2011) Les Activites Informelles et l'occupation des Espaces Publics. Les Trottoirs de lome au Togo. Université de Lomé, Lomé, 12 p.

[41] Steck, J.-F. (2006) La rue Africaine, Territoire de l'informel? Flux, 66-67, 73-86. https://doi.org/10.3917/flux.066.0073 